\title{
Evidence of a Nonequilibrium Distribution of Quasiparticles in the Microwave Response of a Superconducting Aluminum Resonator
}

\author{
P. J. de Visser, ${ }^{1,2, *}$ D. J. Goldie, ${ }^{3}$ P. Diener, ${ }^{1}$ S. Withington, ${ }^{3}$ J. J. A. Baselmans, ${ }^{1}$ and T. M. Klapwijk ${ }^{2}$ \\ ${ }^{1}$ SRON Netherlands Institute for Space Research, Sorbonnelaan 2, 3584 CA Utrecht, The Netherlands \\ ${ }^{2}$ Kavli Institute of NanoScience, Faculty of Applied Sciences, Delft University of Technology, \\ Lorentzweg 1, 2628 CJ Delft, The Netherlands \\ ${ }^{3}$ Cavendish Laboratory, Cambridge University, JJ Thomson Avenue, Cambridge CB3 OHE, United Kingdom
}

(Received 17 June 2013; published 28 January 2014)

\begin{abstract}
In a superconductor, absorption of photons with an energy below the superconducting gap leads to redistribution of quasiparticles over energy and thus induces a strong nonequilibrium quasiparticle energy distribution. We have measured the electrodynamic response, quality factor, and resonant frequency of a superconducting aluminium microwave resonator as a function of microwave power and temperature. Below $200 \mathrm{mK}$, both the quality factor and resonant frequency decrease with increasing microwave power, consistent with the creation of excess quasiparticles due to microwave absorption. Counterintuitively, above $200 \mathrm{mK}$, the quality factor and resonant frequency increase with increasing power. We demonstrate that the effect can only be understood by a nonthermal quasiparticle distribution.
\end{abstract}

DOI: 10.1103/PhysRevLett.112.047004

A superconductor can be characterized by the density of states, which exhibits an energy gap due to Cooper pair formation, and the distribution function of the electrons, which in thermal equilibrium is the Fermi-Dirac distribution. When a superconductor is driven by an electromagnetic field, nonlinear effects in the electrodynamic response can occur, which are usually assumed to be due to a change in the density of states, the so-called pair-breaking mechanism [1]. These nonlinear effects can be described along the lines of a current dependent superfluid density $n_{s}(T, j) \propto n_{s}(T)\left[1-\left(j / j_{c}\right)^{2}\right]$, where $j$ is the actual current density, $j_{c}$ the critical current density, and $T$ the temperature. Observations such as the nonlinear Meissner effect [2] and nonlinear microwave conductivity $[3,4]$ can be explained by a broadening of the density of states and a decreased $n_{s}$. The quasiparticles are assumed to be in thermal equilibrium and a Fermi-Dirac distribution $f(E)=$ $1 /\left[\exp \left(E / k_{B} T\right)+1\right]$ is assumed, with $E$ the quasiparticle energy and $k_{B}$ Boltzmann's constant.

Here we demonstrate that a microwave field also has a strong effect on $f(E)$ in the superconductor, and induces a nonlinear response. We present measurements of the electrodynamic response, quality factor, and resonant frequency of an $\mathrm{Al}$ superconducting resonator (at $5.3 \mathrm{GHz}$ ) as a function of temperature and microwave power at low temperatures $T_{c} / 18<T<T_{c} / 3$. The response measurements, complemented with quasiparticle recombination time measurements, are explained consistently by a model based on a microwave-induced nonequilibrium $f(E)$. Redistribution of quasiparticles $[5,6]$ due to microwave absorption [7] has been shown earlier to cause enhancement of the critical current [8], the critical temperature $\left(T_{c}\right)$, and the energy gap [9]. These enhancement effects are most pronounced close to $T_{c}$ and were
PACS numbers: 74.25.nn, 07.57.Kp, 74.40.Gh, 74.78.-w

observed for temperatures $T>0.8 T_{c}$. A representation of gap suppression and gap enhancement is shown in the inset to Fig. 1(b) [8]. The consequences of the redistribution of quasiparticles for the electrodynamic response were only studied theoretically for $T>0.5 T_{c}$ [10]. Redistribution of quasiparticles also explains [11] the microwave power dependent number of quasiparticles in microwave resonators at low temperatures, which we have recently measured [12]. These quasiparticles impose a limit for detectors for astrophysics based on microwave resonators $[13,14]$. Related phenomena have been reported in superconductingnormal metal devices [15], terahertz pulse experiments [16], and holographic superconductivity [17].

To measure the microwave response, microwave resonators were patterned into a $60 \mathrm{~nm}$ thick $\mathrm{Al}$ film, which was sputter deposited on a sapphire substrate. $T_{c}$ was measured to be $1.17 \mathrm{~K}$, from which the energy gap at zero temperature is taken to be $\Delta=1.76 k_{B} T_{c}=177 \mu \mathrm{eV}$. The low temperature resistivity was $0.9 \mu \Omega \mathrm{cm}$. The film was patterned by wet etching into distributed, half-wavelength, coplanar waveguide resonators, which are capacitively coupled to a transmission line. With readout power $P_{\text {read }}$, we will mean the incident microwave power on the through transmission line. The presented measurements were performed on a resonator with a length of $9.84 \mathrm{~mm}$ and a central strip volume of $1770 \mu \mathrm{m}^{3}$ (sample $A$ ). Sample $B$ is similar and will be introduced later. Further details are provided in the Supplemental Material [18]. The halfwavelength geometry was chosen because it has an isolated central strip, which prevents quasiparticle outdiffusion. The samples were cooled in a pulse tube precooled adiabatic demagnetization refrigerator. Care was taken to make the sample stage light-tight as described in Ref. [19], which is crucial to eliminating excess quasiparticles due to stray 


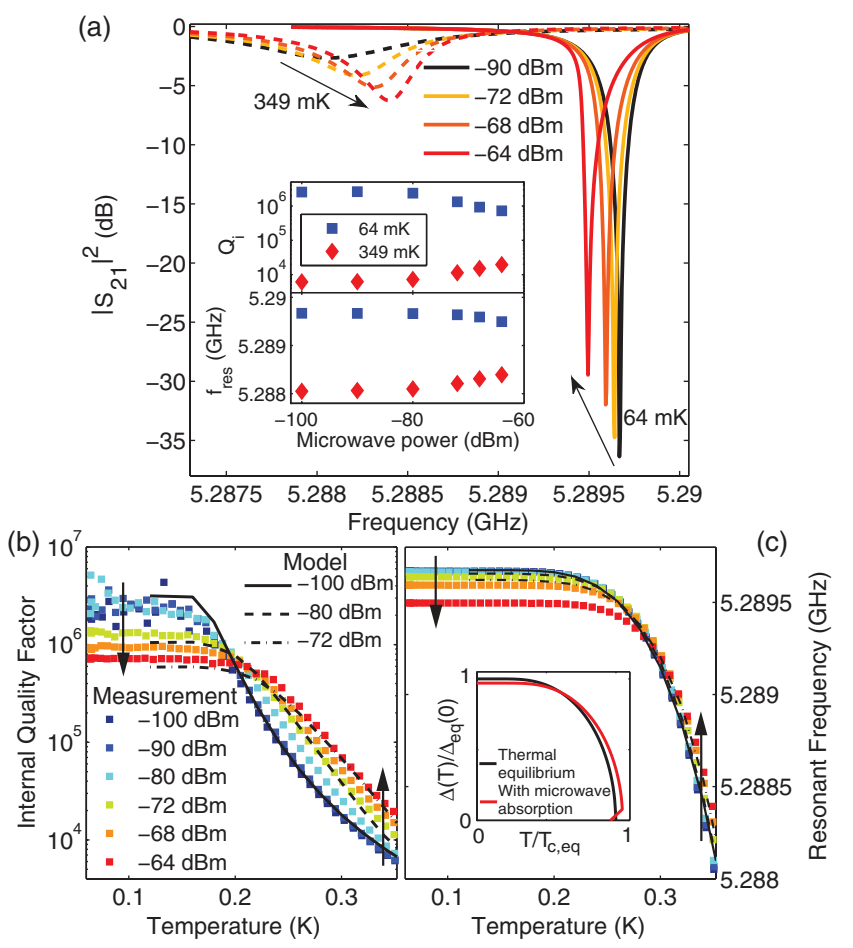

FIG. 1 (color online). (a) The measured microwave transmission $\left|S_{21}\right|^{2}$ as a function of frequency for sample $A$. The solid lines are taken for four different microwave readout powers $\left(P_{\text {read }}\right)$ at a temperature of $64 \mathrm{mK}$. The dashed lines are taken at $349 \mathrm{mK}$. The same color coding applies. The arrows indicate increasing $P_{\text {read }}$. The inset shows the internal quality factor and resonant frequency as determined from the $S_{21}$ measurements as a function of $P_{\text {read }}$. (b),(c) The measured internal quality factor and resonant frequency as a function of temperature for various $P_{\text {read }}$ (the same legend applies). The arrows indicate increasing $P_{\text {read }}$. Simulation results are shown as lines. The inset is a representation of the effect of microwave absorption on the energy gap $\Delta$. The temperature is normalized to the equilibrium $T_{c}$.

light. The complex transmission $S_{21}$ of the microwave circuit was measured with a vector network analyzer. The microwave signal was amplified at $4 \mathrm{~K}$ with a high electron mobility transistor amplifier and with a room temperature amplifier.

We have measured the microwave transmission $S_{21}$ for various $P_{\text {read }}$ as a function of temperature. A selection of resonance curves is shown in Fig. 1(a). We kept $P_{\text {read }}$ below the bifurcation regime [20,21]. By fitting a Lorentzian curve to the resonance curve, we extracted the resonant frequency $\left(f_{\text {res }}\right)$ and the internal quality factor $\left(Q_{i}\right)$ [18], which are plotted for 64 and $349 \mathrm{mK}$ as a function of $P_{\text {read }}$ in the inset in Fig. 1(a) [22]. $Q_{i}$ is higher when the resonance curve is deeper. $Q_{i}$ and $f_{\text {res }}$ are shown for several microwave powers as a function of temperature in Figs. 1(b) and 1(c). Two distinct regimes appear. At low temperatures both $Q_{i}$ and $f_{\text {res }}$ decrease with increasing microwave power, which is consistent with a higher effective electron temperature. At the highest temperatures, however, both $Q_{i}$ and $f_{\text {res }}$ increase with increasing power, which contradicts with a heating model [20] and also cannot be explained by a pair-breaking effect where the density of states broadens due to the current [23]. The pairbreaking mechanism would induce a downward frequency shift without dissipation [4] and might play a role at the highest $P_{\text {read }}$ at the lowest temperatures.

We have modeled the effect of absorption of microwave photons on the quasiparticle distribution function $f(E)$ by using a set of kinetic equations. Absorption of a microwave photon with energy $\hbar \omega$ causes quasiparticles at an energy $E$ to move to an energy $E+\hbar \omega$. The rate with which quasiparticles at energy $E$ absorb photons with energy $\hbar \omega$ can be described with an injection term $I_{\mathrm{qp}}(E, \omega)$ [7], which is given by

$$
\begin{aligned}
I_{\mathrm{qp}}(E, \omega)= & 2 B\left[h_{1}(E, E+\hbar \omega)(f(E+\hbar \omega)-f(E))\right. \\
& \left.-h_{1}(E, E-\hbar \omega)(f(E)-f(E-\hbar \omega))\right],
\end{aligned}
$$

with $h_{1}\left(E, E^{\prime}\right)=\left(1+\left(\Delta^{2} / E E^{\prime}\right)\right) \rho\left(E^{\prime}\right) \cdot \rho(E)$ is the density of states, which is given by $\rho(E)=E / \sqrt{E^{2}-\Delta^{2}}$. B relates the injection rate to the microwave field strength $[11,24]$. The thus created change in $f(E)$ is counteracted by electron-phonon scattering and quasiparticle recombination, which depend both on $f(E)$ and on $n(\Omega)$, the phonon distribution in the film ( $\Omega$ is the phonon energy). In steady state the microwave power that is absorbed by the quasiparticle system is transported through the phonon system of the film and is released in the phonon system of the substrate, the heat bath. We solve the full nonlinear kinetic equations as presented in Ref. [5], together with Eq. (1), in steady state $d f(E) / d t=d n(\Omega) / d t=0$ for all energies, with a self-consistency equation for $\Delta$, given by

$$
\frac{1}{N_{0} V_{\mathrm{BCS}}}=\int_{\Delta}^{\Omega_{D}} \frac{1-2 f(E)}{\sqrt{E^{2}-\Delta^{2}}} d E
$$

with $N_{0}$ the single spin density of states at the Fermi level, $\Omega_{D}$ the Debye energy, and $V_{\mathrm{BCS}}$ the effective pairing potential. The numerical procedure is explained in Ref. [11].

The complex conductivity $\sigma=\sigma_{1}-i \sigma_{2}$, describing the response of both Cooper pairs and quasiparticles to a timevarying electric field with $\hbar \omega<2 \Delta$, is given by [25]

$$
\begin{aligned}
& \frac{\sigma_{1}}{\sigma_{N}}(\omega)=\frac{2}{\hbar \omega} \int_{\Delta}^{\infty}[f(E)-f(E+\hbar \omega)] g_{1}(E) d E, \\
& \frac{\sigma_{2}}{\sigma_{N}}(\omega)=\frac{1}{\hbar \omega} \int_{\Delta-\hbar \omega}^{\Delta}[1-2 f(E+\hbar \omega)] g_{2}(E) d E,
\end{aligned}
$$

where $g_{1}(E)=h_{1}(E, E+\hbar \omega) \rho(E)$ and $g_{2}(E)=h_{1}(E, E+$ $\hbar \omega) E / \sqrt{\Delta^{2}-E^{2}} . \sigma_{N}$ is the normal-state conductivity and 
$\omega$ the angular frequency. Equations (3) and (4) show the role of $f(E)$ in determining the conductivity. In a microwave resonator $f_{\text {res }}$ is proportional to the imaginary part of the conductivity $\sigma_{2}$, and $Q_{i}$ is proportional to $\sigma_{2} / \sigma_{1}$, which connects these observables to $f(E)$.

Since $I_{\mathrm{qp}}$ is proportional to the field strength squared, we need to know the microwave field in the resonator for a certain $P_{\text {read }}$. We solve this problem by using the absorbed microwave power in the quasiparticle system $P_{\mathrm{abs}}$. For the experiment $P_{\text {abs }}$ can be calculated by

$$
P_{\mathrm{abs}}=\frac{P_{\mathrm{read}}}{2} \frac{4 Q^{2}}{Q_{i} Q_{c}} \frac{Q_{i}}{Q_{i, \mathrm{qp}}} .
$$

The loaded quality factor $Q$ is given by $Q=Q_{i} Q_{c} /\left(Q_{c}+\right.$ $\left.Q_{i}\right)$ and $Q_{c}$ is the coupling quality factor. $Q_{c}=\pi /$ $\left(\omega C_{g} Z_{0}\right)^{2}$, with $C_{g}$ the coupling capacitance and $Z_{0}$ the characteristic impedance of the transmission line. See the Supplemental Material [18] for a derivation. Since $Q_{i}$ depends strongly on temperature, $P_{\text {abs }}$ is more than an order of magnitude higher at $300 \mathrm{mK}$ (where $Q_{i}=Q_{c}$ ) than at $100 \mathrm{mK}$ [18], which is a crucial ingredient to model the measurements in Fig. 1 . The factor $Q_{i} / Q_{i, \mathrm{qp}}$ in Eq. (5) arises when $Q_{i}$ is not limited by quasiparticle dissipation. Here we take $Q_{i} / Q_{i, \mathrm{qp}}=1$. $P_{\text {abs }}$ is calculated per unit volume, where the volume is taken to be twice that of the central strip of the resonator, to roughly account for the ground plane of the waveguide, in which power will be absorbed as well. In the calculations we adjust the constant $B$ in Eq. (1), such that $P_{\mathrm{abs}}=4 N_{0} \int_{\Delta}^{\infty} I_{\mathrm{qp}} E \rho(E) d E$.

The simulations were performed for a frequency of 5.57 GHz. The resulting nonequilibrium quasiparticle distributions are shown in Fig. 2(a) for three readout powers for temperatures of 120 and $320 \mathrm{mK}$. A structure with sharp peaks at multiples of $\hbar \omega / \Delta$ shows up due to microwave photon absorption. At $120 \mathrm{mK}$, the driven distribution exceeds the thermal distribution at the bath temperature for all energies, meaning that excess quasiparticles are created. At $320 \mathrm{mK}$, the number of quasiparticles only increases a little at higher power, but quasiparticles are taken away from energies $\Delta<E<\Delta+\hbar \omega$.

In Fig. 2(b) we show the corresponding phonon power flow to the heat bath: $d P(\Omega)=3 N_{\text {ion }} D(\Omega) \Omega[n(\Omega)-$ $\left.n_{\text {sub }}\left(\Omega, T_{\text {bath }}\right)\right] / \tau_{\text {esc }}$. The phonons in the film have a nonequilibrium distribution $n(\Omega)$. Phonons can escape to the substrate, the bath. The phonon distribution in the substrate $n_{\text {sub }}(\Omega)$ is assumed to have a Bose-Einstein distribution at the bath temperature $T_{\text {bath }} \cdot \tau_{\text {esc }}=0.17 \mathrm{~ns}$ is the phonon escape time, calculated for $\mathrm{Al}$ on sapphire using the acoustic mismatch model [26]. $N_{\text {ion }}$ is the number of ions per unit volume and $D(\Omega)=3 \Omega^{2} / \Omega_{D}^{3}$ is the phonon density of states. Figure 2(b) shows strong nonequilibrium behavior as well, with peaks at multiples of $\hbar \omega$. Phonons at $\Omega<2 \Delta$ arise due to scattering. At energies $\Omega>2 \Delta$
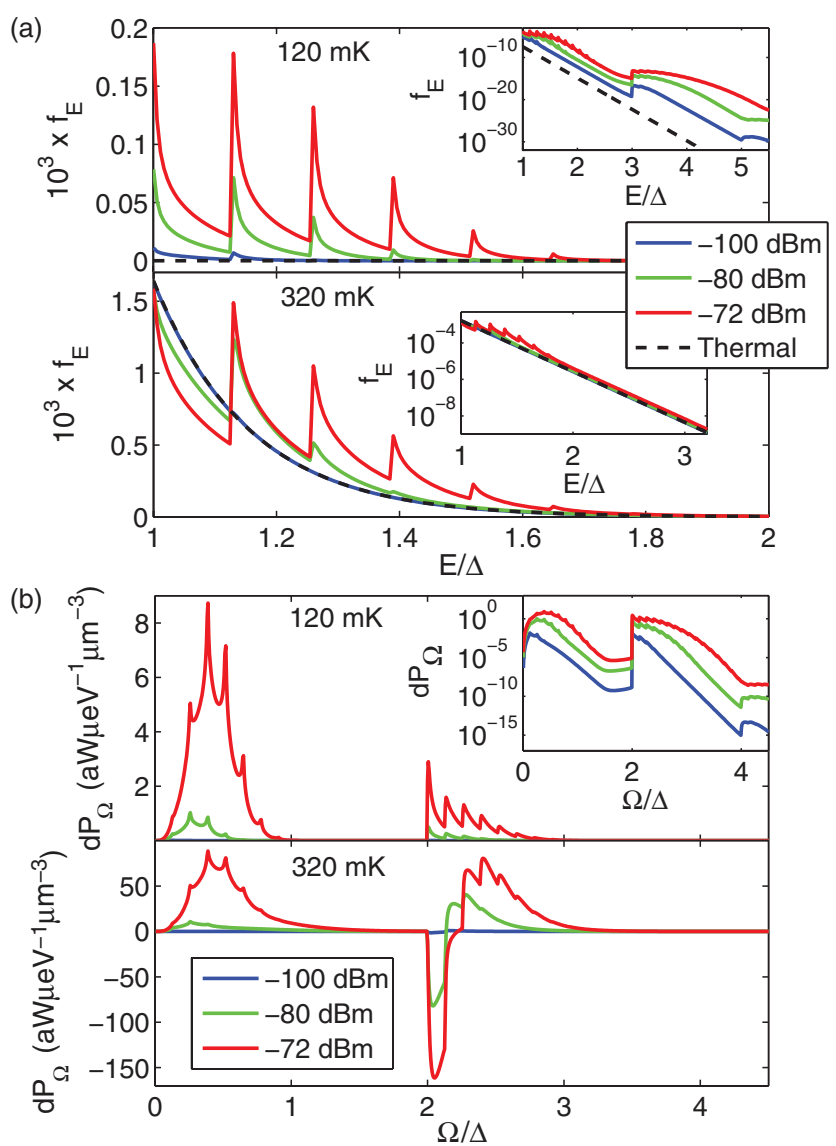

FIG. 2 (color online). (a) The calculated quasiparticle distribution as a function of normalized energy. The two different panels are for temperatures of 120 and $320 \mathrm{mK}$. The insets show the same distributions on a log scale. (b) The phonon power flow from the film to the substrate as a function of normalized energy, for $120 \mathrm{mK}$ (the inset shows the same lines on a log scale) and $320 \mathrm{mK} . d P$ is zero in thermal equilibrium.

phonons due to both recombination and scattering occur. At $320 \mathrm{mK}$, we observe phonon transport out of the film, but also into the film $[d P(\Omega)<0$ at energies $\Omega>2 \Delta]$. This is a consequence of the depletion of $f(E)$ for energies $\Delta<E<\Delta+\hbar \omega$ [Fig. 2(a)] [27].

Having determined the quasiparticle distributions for various readout powers, we can calculate the nonequilibrium conductivity. Figures 3(a) and 3(b) show $\sigma_{1}$ and $\sigma_{2}$, calculated using Eqs. (3) and (4). For comparison, we plot the quasiparticle density and the quasiparticle recombination time $\tau_{\mathrm{qp}}$ in Figs. 3(c) and 3(d). At low temperature, we observe that $\sigma_{1}$ increases and $\sigma_{2}$ decreases with increasing power, together with an increasing number of quasiparticles (analogous to heating), as described in Ref. [11]. At higher temperatures a counterintuitive effect occurs: $\sigma_{1}$ decreases (the microwave losses go down) and $\sigma_{2}$ increases with increasing power, whereas there are still excess quasiparticles being created. This effect cannot be consistently explained with a single effective quasiparticle temperature, but it can be understood from Fig. 2(a) [at 320 mK]. For a 


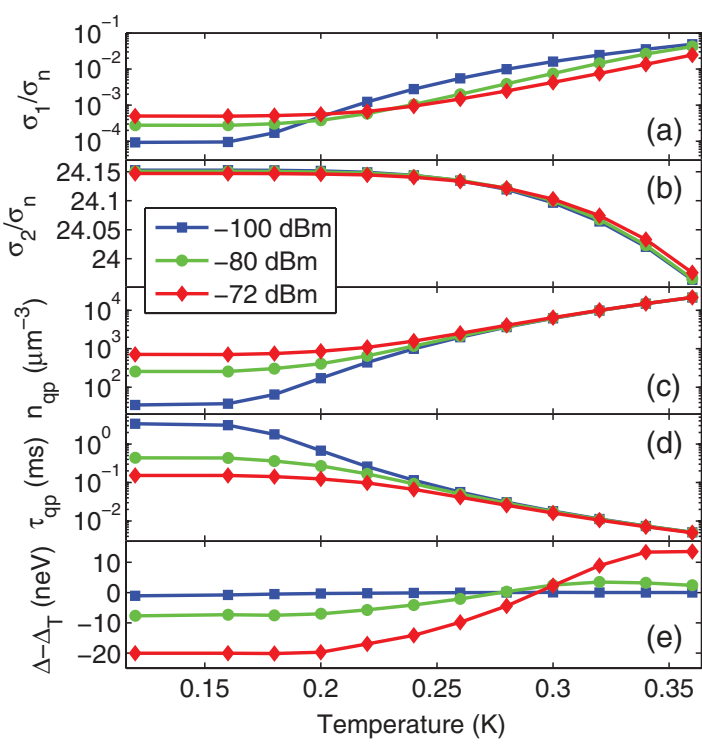

FIG. 3 (color online). (a) The real part of the complex conductivity, $\sigma_{1}$, as a function of temperature, calculated for three microwave readout powers at a frequency of $5.57 \mathrm{GHz}$. (b) The imaginary part of the conductivity, $\sigma_{2}$, as a function of temperature. (c) The calculated quasiparticle density as a function of temperature. (d) The quasiparticle recombination lifetime as function of temperature. (e) The difference of the energy gap for the driven distributions $(\Delta)$ compared to a thermal distribution $\left(\Delta_{T}\right)$. The legend applies to all panels.

thermal $f(E)$, the factor $[f(E)-f(E+\hbar \omega)]$ in Eq. (3) is larger than for a strongly driven distribution, because of the peaks in the driven distribution with separation $\hbar \omega$. The probability of absorbing a microwave photon is lower for a strongly driven distribution, which decreases $\sigma_{1}$ and therewith the losses. $\sigma_{2}$ is only sensitive to quasiparticles at $\Delta<E<\Delta+\hbar \omega$ [Eq. (4)]. Below $250 \mathrm{mK}$ [see Fig. 3(b)], the microwave absorption increases the quasiparticle population at $\Delta<E<\Delta+\hbar \omega$, whereas at higher temperatures the population becomes lower due to redistribution. The energy gap, calculated from Eq. (2), is shown in Fig. 3(e). Clearly, the nonequilibrium $f(E)$ leads to gap suppression below $0.3 \mathrm{~K}$, and gap enhancement above $0.3 \mathrm{~K}$ despite the creation of excess quasiparticles. The additional effect of the nonequilibrium $\Delta$ on the observables is minor, the structure in $f(E)$ dominates.

To connect the calculated $\sigma_{1}$ and $\sigma_{2}$ with the experiment, we calculate $Q_{i}$ and $f_{\text {res }}$ through equations for a microstrip geometry [28] with the same central strip dimensions as the measured resonator [18]. The results are plotted in Figs. 1(b) and 1(c), which show good agreement with the measurements. In particular, the crossover temperatures in $Q_{i}$ and $f_{\text {res }}$ are well modeled, as is the temperature dependence of $Q_{i}$ for both high and low powers. A comparison of Figs. 1(b) and 1(c) with Figs. 3(a) and 3(b) shows that $Q_{i}$ is dominated by $\sigma_{1}$ and $f_{\text {res }}$ by $\sigma_{2}$, as expected.

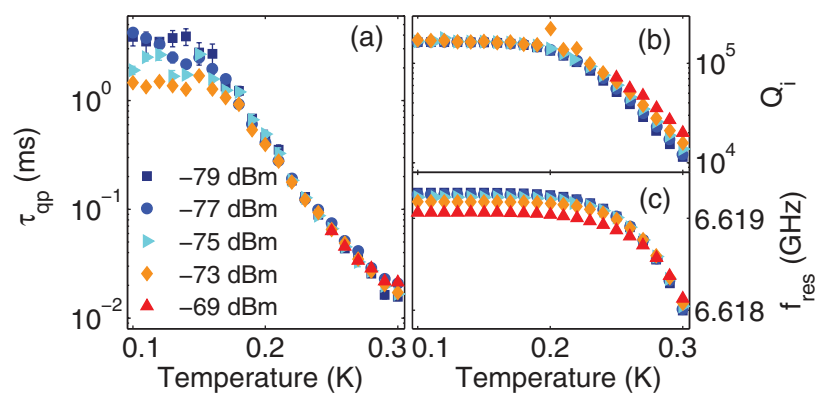

FIG. 4 (color online). (a) The measured quasiparticle recombination time as obtained from a noise measurement for various microwave readout powers measured on sample $B$. (b) The measured internal quality factor and (c) resonant frequency. The legend applies to all panels.

The experimental evidence for the different power dependence of $\tau_{\mathrm{qp}}$ and the conductivity is shown in Fig. 4. These results were measured on sample $B$ [18], on which we performed accurate measurements of $\tau_{\mathrm{qp}}$ as reported on in Refs. [12,29]. Figure 4(a) shows $\tau_{\mathrm{qp}}$ as determined from the cross-power spectral density of quasiparticle fluctuations in the amplitude and the phase of the resonator [12]. Panels (b) and (c) show the measured $Q_{i}$ and $f_{\text {res. }}$. The power range for this noise measurement is only $10 \mathrm{~dB}$, due to the amplifier noise limit. We focus on $T>200 \mathrm{mK} . Q_{i}$ increases with increasing power, consistent with Fig. 1(b), whereas $\tau_{\mathrm{qp}}$ stays constant, as expected from the simulations in Fig. 3(d). We thus have a nonlinear conductivity effect due to quasiparticle redistribution, where $Q_{i}$ increases despite the creation of excess quasiparticles. This is in contrast with situations in which excess quasiparticles are introduced either on purpose or due to the environment [30-36] where $Q_{i} \propto 1 / n_{\mathrm{qp}}$, although also in qubits subtleties can occur due to $f(E)$ [37].

The qualitative agreement between measurements and calculations as apparent from Fig. 1(b) is quite satisfactory. However, the effect of the microwave power on $Q_{i}$ and $f_{\text {res }}$ is less than calculated. Since the uncertainty in the measured $P_{\text {read }}$ is less than $2 \mathrm{~dB}$, there should be a parallel dissipation channel. So far we assumed the same $f(E)$ for the ground plane of the resonator and the central strip. Future work may include the calculation of $f(E)$ in the ground plane, which is difficult due to the additional complexity of quasiparticle outdiffusion. A crude approximation, where the ground plane is an impedance with a thermal $f(E)$, in series with the nonequilibrium central strip [38], indicates indeed a reduced nonequilibrium effect of microwave power on $Q_{i}$ and $f_{\text {res. }}$. The nonequilibrium $f(E)$ could be measured by combining the resonator experiment with tunnel probes [6].

In closing, we emphasize that for the nonequilibrium $f(E)$ to occur [Fig. 2(a)], quasiparticle-phonon scattering has to be slow compared to $I_{\mathrm{qp}}$ and to $\omega$, which is therefore 
more likely in materials with a low $T_{c}$, such as $\mathrm{Al}$ [39]. In addition, redistribution of quasiparticles at low temperatures leads to $n_{\mathrm{qp}} \propto \sqrt{P_{\mathrm{abs}}}$ [11], which implies that even in the few microwave photon regime this mechanism leads to excess quasiparticles.

We would like to thank Y. J. Y. Lankwarden for fabricating the devices. T. M. K. thanks J. Zmuidzinas and A. Vayonakis for discussions on this topic.

*p.j.devisser@tudelft.nl

[1] This pair-breaking mechanism is different from direct Cooper pair breaking by, e.g., photons.

[2] S. K. Yip and J. A. Sauls, Phys. Rev. Lett. 69, 2264 (1992).

[3] C. C. Chin, D. E. Oates, G. Dresselhaus, and M. S. Dresselhaus, Phys. Rev. B 45, 4788 (1992).

[4] B. H. Eom, P. K. Day, H. G. LeDuc, and J. Zmuidzinas, Nat. Phys. 8, 623 (2012).

[5] J.-J. Chang and D. J. Scalapino, Phys. Rev. B 15, 2651 (1977).

[6] J. Wolter and R. E. Horstman, Phys. Lett. 86A, 185 (1981).

[7] G. M. Eliashberg, JETP Lett. 11, 114 (1970); B. I. Ivlev, S. G. Lisitsyn, and G. M. Eliashberg, J. Low Temp. Phys. 10, 449 (1973).

[8] T. M. Klapwijk and J.E. Mooij, Physica (Amsterdam) 81B+C, 132 (1976); T. M. Klapwijk, J. N. van den Bergh, and J. E. Mooij, J. Low Temp. Phys. 26, 385 (1977).

[9] R. E. Horstman and J. Wolter, Phys. Lett. 82A, 43 (1981).

[10] S. Sridhar and J.E. Mercereau, Phys. Lett. 75A, 392 (1980).

[11] D. J. Goldie and S. Withington, Supercond. Sci. Technol. 26, 015004 (2013).

[12] P. J. de Visser, J. J. A. Baselmans, S. J. C. Yates, P. Diener, A. Endo, and T. M. Klapwijk, Appl. Phys. Lett. 100, 162601 (2012).

[13] P. K. Day, H. G. LeDuc, B. A. Mazin, A. Vayonakis, and J. Zmuidzinas, Nature (London) 425, 817 (2003).

[14] J. Zmuidzinas, Annu. Rev. Condens. Matter Phys. 3, 169 (2012).

[15] F. Chiodi, M. Aprili, and B. Reulet, Phys. Rev. Lett. 103, 177002 (2009); P. Virtanen, T. T. Heikkilä, F. S. Bergeret, and J. C. Cuevas, Phys. Rev. Lett. 104, 247003 (2010).
[16] M. Beck, I. Rousseau, M. Klammer, P. Leiderer, M. Mittendorff, S. Winnerl, M. Helm, G. N. Gol'tsman, and J. Demsar, Phys. Rev. Lett. 110, 267003 (2013).

[17] N. Bao, X. Dong, E. Silverstein, and G. Torroba, J. High Energy Phys. 10 (2011) 123.

[18] See Supplemental Material at http://link.aps.org/ supplemental/10.1103/PhysRevLett.112.047004 for material and device parameters and a derivation of the absorbed microwave power in the quasiparticle system.

[19] J. Baselmans, S. Yates, P. Diener, and P. de Visser, J. Low Temp. Phys. 167, 360 (2012).

[20] P. J. de Visser, S. Withington, and D. J. Goldie, J. Appl. Phys. 108, 114504 (2010).

[21] L. J. Swenson, P. K. Day, B. H. Eom, H. G. Leduc, N. Llombart, C. M. McKenney, O. Noroozian, and J. Zmuidzinas, J. Appl. Phys. 113, 104501 (2013).

[22] At $64 \mathrm{mK}$, a $P_{\text {read }}$ of $-64(-100) \mathrm{dBm}$ leads to a stored energy of $0.55 \mathrm{fJ}(0.11 \mathrm{aJ})$, corresponding to $1.6 \times$ $10^{8}\left(3.1 \times 10^{4}\right)$ photons.

[23] S. B. Nam, Phys. Rev. 156, 470 (1967)

[24] J. E. Mooij and T. M. Klapwijk, Phys. Rev. B 27, 3054 (1983).

[25] D. C. Mattis and J. Bardeen, Phys. Rev. 111, 412 (1958).

[26] S. B. Kaplan, J. Low Temp. Phys. 37, 343 (1979).

[27] A. M. Gulian and G. F. Zharkov, J. Low Temp. Phys. 48, 125 (1982).

[28] G. Yassin and S. Withington, J. Phys. D 28, 1983 (1995).

[29] P. J. de Visser, J. J. A. Baselmans, P. Diener, S. J. C. Yates, A. Endo, and T. M. Klapwijk, Phys. Rev. Lett. 106, 167004 (2011).

[30] J. Gao, J. Zmuidzinas, A. Vayonakis, P. Day, B. Mazin, and H. Leduc, J. Low Temp. Phys. 151, 557 (2008).

[31] J. M. Martinis, M. Ansmann, and J. Aumentado, Phys. Rev. Lett. 103, 097002 (2009).

[32] G. Catelani, L. I. Glazman, and K. E. Nagaev, Phys. Rev. B 82, 134502 (2010).

[33] A. D. Córcoles, J. M. Chow, J. M. Gambetta, C. Rigetti, J. R. Rozen, G. A. Keefe, M. B. Rothwell, M. B. Ketchen, and M. Steffen, Appl. Phys. Lett. 99, 181906 (2011).

[34] R. Barends et al., Appl. Phys. Lett. 99, 113507 (2011).

[35] M. Lenander et al., Phys. Rev. B 84, 024501 (2011).

[36] D. Rainis and D. Loss, Phys. Rev. B 85, 174533 (2012).

[37] J. Wenner et al., Phys. Rev. Lett. 110, 150502 (2013).

[38] R. L. Kautz, J. Appl. Phys. 49, 308 (1978).

[39] S. B. Kaplan, C. C. Chi, D. N. Langenberg, J. Chang, S. Jafarey, and D. J. Scalapino, Phys. Rev. B 14, 4854 (1976). 